\title{
Electrochemotherapy in treatment of canine oral malignant melanoma and factors influencing treatment outcome
}

\author{
Matías Nicolás Telladoํㅜ, Felipe Horacio Maglietti², Sebastián Diego Michinski³ \\ Guillermo Ricardo Marshall ${ }^{3}$, Emanuela Signori ${ }^{4}$ \\ VetOncologia Cancer Clinic, Ciudad de Buenos Aires, Argentina \\ ${ }^{2}$ Instituto Universitario del Hospital Italiano - CONICET, Ciudad de Buenos Aires, Argentina \\ ${ }^{3}$ Instituto de Física del Plasma, Departamento de Física, FCEyN, Universidad de Buenos Aires - CONICET, \\ Ciudad de Buenos Aires, Argentina \\ ${ }^{4}$ CNR-Institute of Translational Pharmacology, Roma, Italy
}

Radiol Oncol 2020; 54(1): 68-78.

Received 31 December 2019

Accepted 23 February 2020

Correspondence to: Felipe Horacio Maglietti, Instituto Universitario del Hospital Italiano - CONICET, Ciudad de Buenos Aires, C1199ACL, Argentina. E-mail: felipemaglietti@gmail.com

Matías Nicolás Tellado and Felipe Horacio Maglietti contributed equally to this work.

Disclosure: No potential conflicts of interest were disclosed.

Background. Oral malignant melanoma is the most common, but aggressive oral cancer in dogs with poor prognosis. Electrochemotherapy (ECT) has therapeutic potential in such tumors as effective local treatment. Therefore, the aim of this prospective clinical study was to evaluate treatment effectiveness of ECT in as first line treatment for canine oral malignant melanoma, and search for factors influencing treatment outcome.

Methods. Sixty-seven canines with primary oral malignant melanoma, non-candidates for first-line therapy, were enrolled. All dogs received ECT and follow-up exams for the span of two years.

Results. Based on RECIST criteria, the objective response rate was $100 \%, 89.5 \%, 57.7 \%$, and $36.4 \%$, in stage I, II, III and IV, respectively. Only patients in stage I, II and III with partial or complete response improved their quality of life. The median time to progression was 11, 7, 4 and 4 months, and median survival time after the treatment was 16.5, 9.0, 7.5 and 4.5 months, for patients in stage I, II, III and IV, respectively. Significantly better was local response in stage I and II disease $(p=0.0013)$, without the bone involvement $(p=0.043)$

Conclusions. Electrochemotherapy is effective local treatment of oral canine malignant melanoma when no alternative treatment is available. Better response is expected in stage I and II patients with tumors without bone involvement.

Key words: cancer; dog; electroporation; electrochemotherapy

\section{Introduction}

Oral malignant melanoma represents $6 \%$ of neoplasms in canines, being the most common oral cancer among that species. It is more aggressive and has a poorer prognosis than any other melanoma. ${ }^{1,2}$ The mean age at diagnosis is 11.6 years. ${ }^{3}$

Most common breeds affected by melanoma are Cocker Spaniel, German Shepherd, Pointer, Weimaraner, Golden Retriever, Labrador
Retriever, Poodle, Chow-Chow and Boxer. It usually infiltrates locally, with metastatic progression in more than $80 \%$ of the cases. Typically, there is a better outcome and prognosis with early diagnosis and/or rostral location. On the other hand, worse prognosis is associated with late diagnosis, caudal location, presence of satellite lesions, and when dysphagia and dyspnea are present. ${ }^{4}$

It is known that lack of treatment is associated with poor survival times (average of 65 days)2, 
while best survival times are achieved with clean margins at surgical excision plus radiotherapy. Therefore, the latter is considered the first-line treatment for this neoplasm, with a recurrence of distant metastasis that can arise 4 to 8 months after the end of it. Given the fact that most of the tumors in advanced stages are already involving bony structures, complete surgical resection is often very aggressive and has poor prognosis. Adjuvant chemotherapy does not increase response rates or adds any survival benefit to the patients., 6

Electrochemotherapy (ECT) is a therapeutic modality that has been gaining ground in Oncology since the Standard Operating Procedures were published in 2006. ${ }^{7}$ ECT consists in the application of an electric field to a tumor in order to increase the uptake of bleomycin that was previously administered (locally or intravenously) at a very low dose $^{8,9}$; alternatively, cisplatin can be used locally with equally good results. ${ }^{10}$ ECT is primarily indicated for cutaneous and subcutaneous tumors of any histology. After the electric field is applied, a cell membrane permeabilization is produced by a physical phenomenon known as electroporation, affecting all tumor cells, regardless of the histological tissue. ${ }^{11}$ A meta-analysis of ECT clinical studies in human oncology showed that the overall objective response (OR) rate varies from $62.6 \%$ to $82.2 \%$ depending also on which route was used to administer the drug. ${ }^{12}$ Great efforts are being made to extend ECT to non-cutaneous locations such as liver ${ }^{13}$, brain ${ }^{14}$, and bones. ${ }^{15}$ Moreover, an endoscopic electrode was developed for treating the colon. ${ }^{16}$ The considerable experience and deeper understanding gained since ECT inception, lead to the publication of new and more flexible Standard Operating Procedures. ${ }^{17}$ In veterinary medicine, ECT is a well-established therapy with multiple indications and proven efficacy. ${ }^{18,19}$ It has been reported very good results for treating mast-cell tumors (OR 62.3-100\%) ${ }^{20-22}$, sarcomas (OR 90\%) ${ }^{23}$, perianal tumors (OR 94\%) ${ }^{24}$, nasal duct tumors (OR $91 \%)^{24,25}$, among others, with minimum to absent side effects.

In vivo, ECT is remarkably more effective in immunocompetent subjects. ${ }^{26}$ Different mechanisms for the induced local immune response have been demonstrated in preclinical studies. Some of them have also been observed in human patients. However, this local immune response does not induce an abscopal effect, and intense efforts are being made in order to achieve it. 27,28

The clinical experience with melanoma immunotherapies seems promising, with increasing evi- dence that combined approaches may be required to ensure durable responses in the majority of the patients. ${ }^{29}$

In this prospective clinical study, we present results obtained from 67 canine patients affected by oral malignant melanomas that were treated with ECT and later followed-up for up to two years. We also discuss ECT benefits in comparison to classic therapeutic approaches and search for factors influencing treatment outcome.

\section{Methods}

In this longitudinal prospective study, sixty-seven (67) canine (Canis familiaris) patients with oral malignant melanoma and not candidates for first-line therapy were enrolled. Primary goals consisted on evaluating local response and survival times. In addition, we also decided to evaluate quality of life, time to progression and disease-free survival times. Treatments were performed from 10/2014 through 12/2017 at Centro de Especialidades Medico Veterinarias, Buenos Aires, Argentina.

Inclusion criteria:

- Patients with oral malignant melanoma at any stage.

- Patients able to stand general anesthesia.

Exclusion criteria:

- Pregnant females

- Patients are candidates for surgery and owner agrees to perform treatment.

\section{Patient evaluation}

Participants' diagnosis was confirmed by histopathology (surgical biopsies). Immunohistochemistry was performed in selected cases for amelanotic melanoma or when diagnosis was not definitive.

Patients were staged according to the WHO staging system for canine oral melanoma (Table 1). ${ }^{30}$ They underwent a complete physical examination, complete blood count (CBC), and serum biochemi-

TABLE 1. WHO criteria for staging canine oral melanoma

\begin{tabular}{llll}
\cline { 1 - 2 } Stage & Tumor diameter & $\begin{array}{l}\text { Lymph node } \\
\text { involvement }\end{array}$ & Metastasis \\
\cline { 1 - 2 } I & $<2 \mathrm{~cm}$ & No & No \\
II & $2-4 \mathrm{~cm}$ & No & No \\
III & $>$ or $=4 \mathrm{~cm}$ & No & No \\
& Any & Yes & No \\
IV & Any & Yes or No & Yes \\
\hline
\end{tabular}




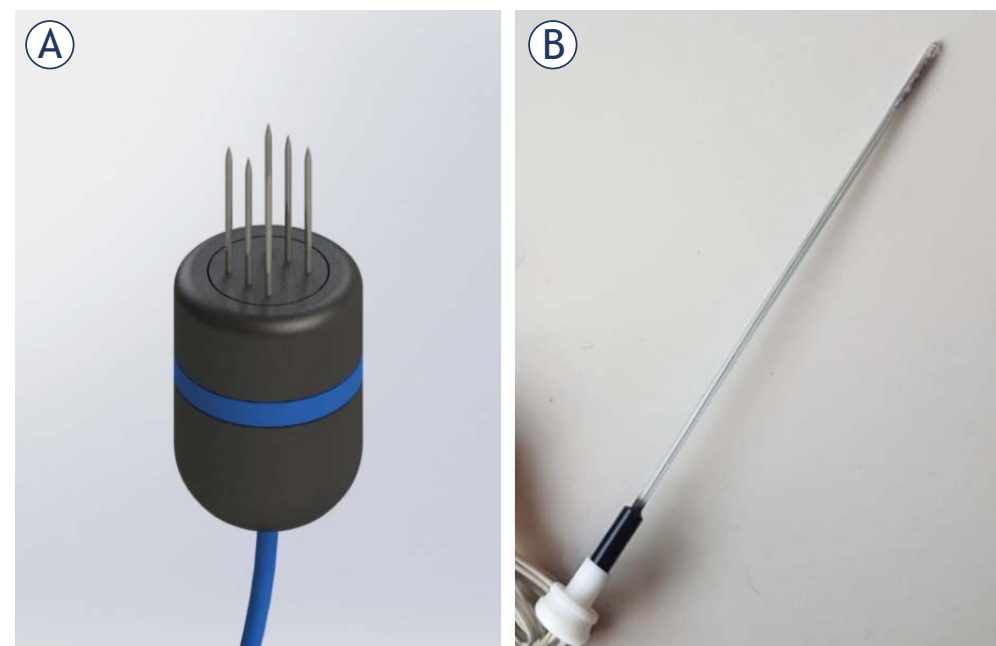

FIGURE 1. Electrodes used to treat patients. In (A) a render of the six-needle electrode used. It consists of two rows of needles separated $4 \mathrm{~mm}$ from each other In (B) a picture of the Single Needle Electrode for nasal duct treatment.

cal analysis. The size of the tumor was measured using a caliper or by CT scan for masses involving deeper structures such as retro-orbital region, nasopharynx or nasal cavity. In addition, to confirm or rule out bone involvement when plain radiographs were not fully diagnostics.

Evaluation for metastatic disease was performed via 3-view thoracic radiographs, abdominal ultrasound and fine-needle aspirate of regional lymph nodes. CT scan was performed when radiographs were not diagnostics. Data gathered in all patients included: age, weight, sex, breed, stage at time of diagnosis, histologic subtype, presence or not of bone infiltration, location of the tumor within the mouth (was considered caudal when compromising the caudal third of the hard palate, soft palate, oropharynx, angle of the mandible, ramus of the mandible or the tonsillar region. Otherwise, it was considered to be rostral), local response, quality of life, presence/absence of metastasis, number of ECT sessions, new metastasis after treatment, time to progression, disease-free survival times (when CR was obtained), overall survival times and cause of death.

\section{ECT procedure}

ECT and surgical resection of enlarged regional lymph nodes were performed under general anesthesia.

Chosen anesthesia protocol was proved to provide adequate comfort throughout the procedure, and consisted on premedication with IM (intramus- cular) administration of xylazine (Xylazine $100 \AA$, Richmond, Buenos Aires, Argentina) $0.5 \mathrm{mg} / \mathrm{kg}$ and tramadol (Tramadol®, John Martin, Buenos Aires, Argentina) $2 \mathrm{mg} / \mathrm{kg}$. Induction was performed with IV (intravenous) administration of propofol (Propofol Gemepe®, Gemepe, Buenos Aires, Argentina) $2-3 \mathrm{mg} / \mathrm{kg}$. For maintenance, isoflurane (Zuflax®, Richmond, Buenos Aires, Argentina) $2-3 \%$ and intravenous fentanyl (Fentanyl Gemepe ${ }^{\circledR}$, Gemepe, Buenos Aires, Argentina) 2 $\mathrm{mcg} / \mathrm{kg}$ were used. Amoxicillin with clavulanic acid (Clavamox ${ }^{\circledR}$ Zoetis, Buenos Aires Argentina) $15 \mathrm{mg} / \mathrm{kg} / \mathrm{bid}$ and meloxicam (Meloxivet ${ }^{\circledR}$, John Martin, Buenos Aires, Argentina) $0.2 \mathrm{mg} / \mathrm{kg} / \mathrm{SID}$ were administered orally for prophylaxis and analgesia after the treatment according to the needs of each patient.

ECT was initiated by using IV (intravenous) bleomycin (Blocamicina ${ }^{\circledR}$, Gador, Buenos Aires, Argentina) at a dose of $15000 \mathrm{IU} / \mathrm{m}^{2}$ of body surface. Eight minutes later, in order to allow drug distribution, electric pulses were delivered (following Standard Operating Procedures $)^{17}$ with a BTX ECM 830 (Harvard Apparatus, Holliston, MA, USA) unit. Each train of pulses consisted of eight (8) square wave monopolar pulses of $400 \mathrm{~V}(1000 \mathrm{~V} / \mathrm{cm})$ $100 \mu$ s long at $10 \mathrm{~Hz}$. The number of trains applied varied according to the tumor size, aiming to cover the whole tumor volume plus safety margins beyond it. In all cases, a 6-needle electrode was used (Figure 1A.), but for nasal duct invasion, the Single Needle Electrode ${ }^{\circledR}$ was indicated (Figure 1B.). ${ }^{25}$ This electrode uses the same pulse parameters, but thirty-two (32) instead of eight (8) pulses are delivered on each train. Recommendations to report results in electrochemotherapy studies from Campana et al. were followed. ${ }^{31}$

\section{Patient follow-up}

Patients were scheduled for checkups at 14,30 and 60 days after treatment. If no further sessions were needed, follow-ups were required every 3 to 4 months until the end of the study. On each visit, a complete clinical examination and thoracic radiographs were performed to determine if lymph node enlargement and/or development of lung metastasis respectively were noticed; as they represent the most common sites for metastatic dissemination. ${ }^{6}$

\section{Treatment response evaluation}

According to the RECIST criteria, the response was determined at 30 days and confirmed at 60 days 


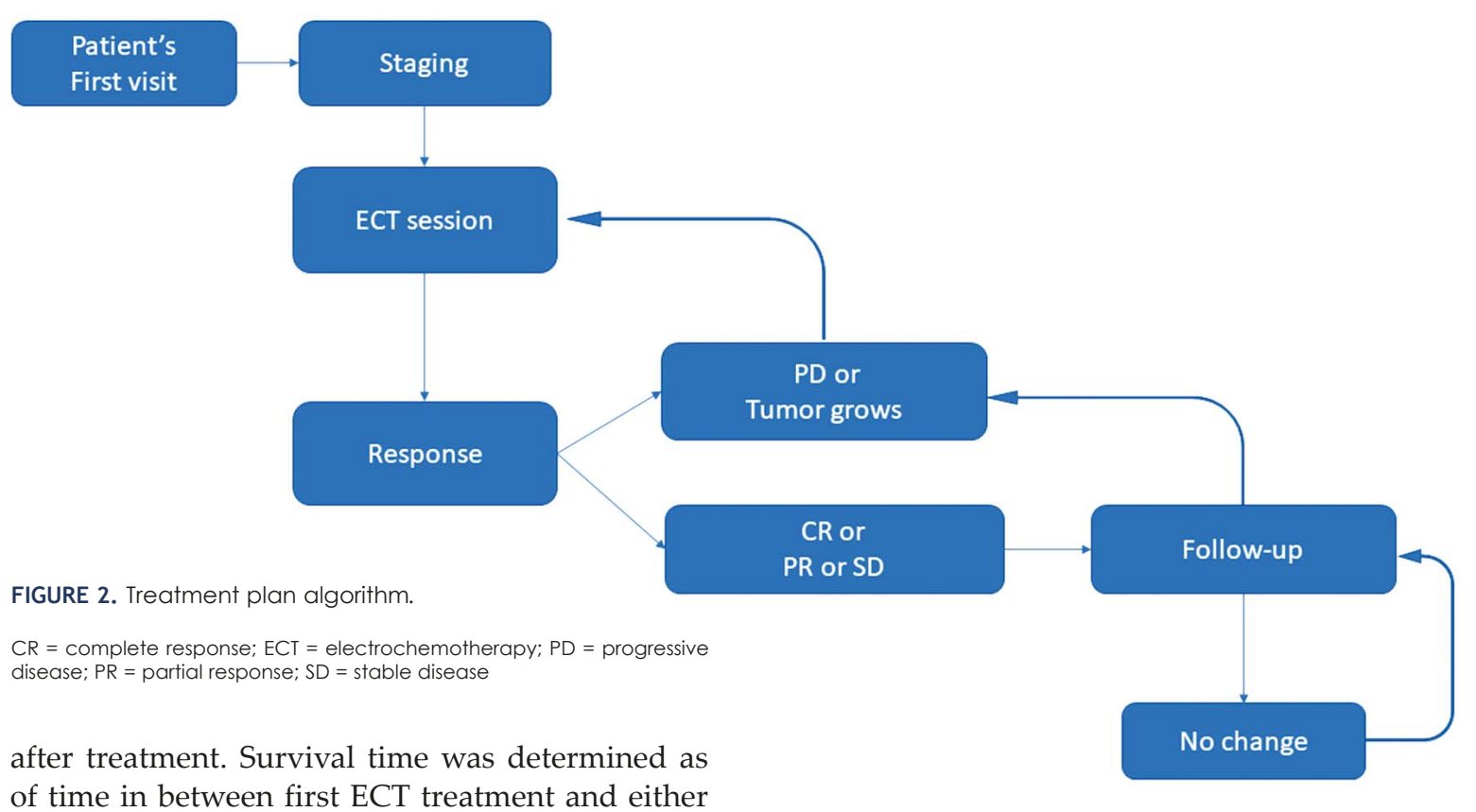
of time in between first ECT treatment and either end of the study or death of the patient. Time to progression was determined to be from the day of the ECT session that achieved a confirmed response through the day of an either local or distant relapse. Disease-free survival time was calculated only among patients that were able to achieve a CR. It was determined as time between a CR was achieved through the day where a relapse, local or distant, was evidenced.

\section{Quality of life}

The quality of life was assessed before the first ECT treatment and by each follow up exam using a questionnaire inspired in the observations of Lynch et al. ${ }^{32}$ It was filled by the owner (provided in additional material). It consisted of three (3) clinical and behavioral questions, with values between 0 and 3 . Final score was obtained by the sum of all questions, with lower scores representing better results (score of 0 ) and higher scores worse quality of life (score of 9). In order to consider if quality of life was either improved or worsened, a difference of at least two (2) units was required when comparing both scores (day of the treatment vs. each follow-up).

Data obtained by the treating oncologist was also included in the patient's medical record.

\section{Criteria for ECT retreatment and additional therapy}

If tumor growth was detected in any given follow-up visit, then a new session was scheduled.

Likewise, if the achieved response was a progressive disease (PD), a new session was performed. Any enlarged regional lymph node noticed by the time of a follow-up (with metastatic spread determined by fine needle aspirate) was surgically removed (Figure 2).

\section{Statistical analysis}

Statistics were performed using MedCalc $®$ version 19.1. Fisher exact test was used to determine the significant association between local response and different factors studied. Multivariate logistic regression analysis was performed with the factors that showed significant association with local response.

Kaplan-Meier curves were calculated, and their significance determined by the log-rank test, for different factors affecting survivorship and time to progression. Cox multiple regression analysis using forward regression method was performed with statistically significant factors for survival.

\section{Ethics approval and consent to participate}

All regulations from the Consejo Profesional de Médicos Veterinarios were followed. Informed consents were signed by the owners. This work was approved by the IACUC of the School of Veterinary Sciences, University of Buenos Aires, Argentina. Protocol number: 2018/31. 




FIGURE 3. The bars show the sum of partial and complete response percentages obtained on each stage. For stage I: complete responses (CR) $72.7 \%$ (8) partial responses (PR) $27.3 \%$ (3) stable diseases (SD) $0 \%$ progressive diseases (PD) $0 \%$, stage II: CR $21.1 \%(4)$ PR $68.4 \%$ (13) SD 0\% PD 10.5\% (2), stage III: CR $7.7 \%$ (2) PR 50\% (13) SD $26.9 \%$ (7) PD 15.4\% (4) and stage IV: CR 0\% PR 36.4\% (4) SD 36.4\% (4) PD 27.3\% (3).

\section{Results}

Demographics of the treated patients is in Table 2. The patients were followed up for two years. Two (2) out of the sixty-seven (67) treated patients remained alive by the end of the study. Sixty-one percent of the patients (41) required a single session to achieve a final response, $30 \%$ (20) required two, 7\% (5) required three and 1\% (1) required four procedures. Time between sessions varied in each case depending on tumoral regrowth, and usually is between one and two months.

\section{Local response}

After a median of 1.5 treatment sessions (regardless of the stage), the overall objective response (OR) rate was $70.1 \%$ (47); with $20.9 \%$ (14) complete responses (CR), 49.3\% (33) partial responses (PR), 16.4\% (11) stable diseases (SD) and 13.4\% (9) progressive diseases (PD). Local response in each stage is presented in Figure 3.

Logistic regression analysis showed that early stages of disease (stages I and II) were associated with significantly higher objective response rates than late stages (III and IV), $93.3 \%$ vs. $51.4 \%$, respectively (HR: $0.08,95 \%$ CI: $0.02-0.36, p=0.0013$ ) (Table 3).

\section{Time to progression and disease-free survival times}

The median time to progression was 11 (range 4-30) months, 7 (range 3-21) months, 4 (range 2-4) months and 4 (range 1-4) months, for stages I, II, III and IV, respectively.
TABLE 2. Patients' demographics

\begin{tabular}{|c|c|}
\hline Characteristics of 67 patients & Mean (range) (\%) \\
\hline Age (years) & $11.7(6-16)$ \\
\hline Sex ratio (M:F) & $37: 30$ \\
\hline Body weight (kg) & $22.6(3.5-58)$ \\
\hline \multicolumn{2}{|l|}{ Breeds } \\
\hline Crossbreed & $24(35.8 \%)$ \\
\hline Labrador retriever & $9(13.4 \%)$ \\
\hline Cocker spaniel & 7 (10.4\%) \\
\hline Golden retriever & $6(8.9 \%)$ \\
\hline Beagle & $4(5.9 \%)$ \\
\hline Poodle & $4(5.9 \%)$ \\
\hline Rottweiler & $3(4.4 \%)$ \\
\hline Doberman & $2(2.9 \%)$ \\
\hline Shar-Pei & $2(2.9 \%)$ \\
\hline Dogo & $1(1.4 \%)$ \\
\hline English Mastiff & $1(1.4 \%)$ \\
\hline Chow-Chow & $1(1.4 \%)$ \\
\hline Basset Hound & $1(1.4 \%)$ \\
\hline Pekingese & $1(1.4 \%)$ \\
\hline Dalmatian & $1(1.4 \%)$ \\
\hline Location (Rostral : Caudal) & $36: 31$ \\
\hline \multicolumn{2}{|l|}{ Stage } \\
\hline 1 & $11(16.5 \%)$ \\
\hline$\|$ & 19 (28.3\%) \\
\hline III & $26(38.8 \%)$ \\
\hline IV & $11(16.4 \%)$ \\
\hline \multicolumn{2}{|l|}{ Histologic subtype } \\
\hline Epithelioid & $17(25.4 \%)$ \\
\hline Mixed & $17(25.4 \%)$ \\
\hline Spindle cell & $17(25.4 \%)$ \\
\hline Anaplastic & $9(13.4 \%)$ \\
\hline Others & $7(10.4 \%)$ \\
\hline
\end{tabular}

The multivariate analysis for the time to progression showed that stages I $(p=0.0005)$ and II $(p=0.0094)$, and absence of bone invasion $(p=0.043)$ were predictive factors for longer times to progression (Table 4).

Considering the patients who achieved a complete response $(n=14)$, the median disease-free survival time for these patients was 12.5 (range 3-30) months. Among them: $2(14 \%)$ remained alive at the end of the study, $6(43 \%)$ died of unrelated causes. Only one (7\%) developed distant metastases during the follow-up, three months after the complete response was obtained. Twelve (86\%) were treated with only one session of ECT. Four (29\%) had bone involvement. 

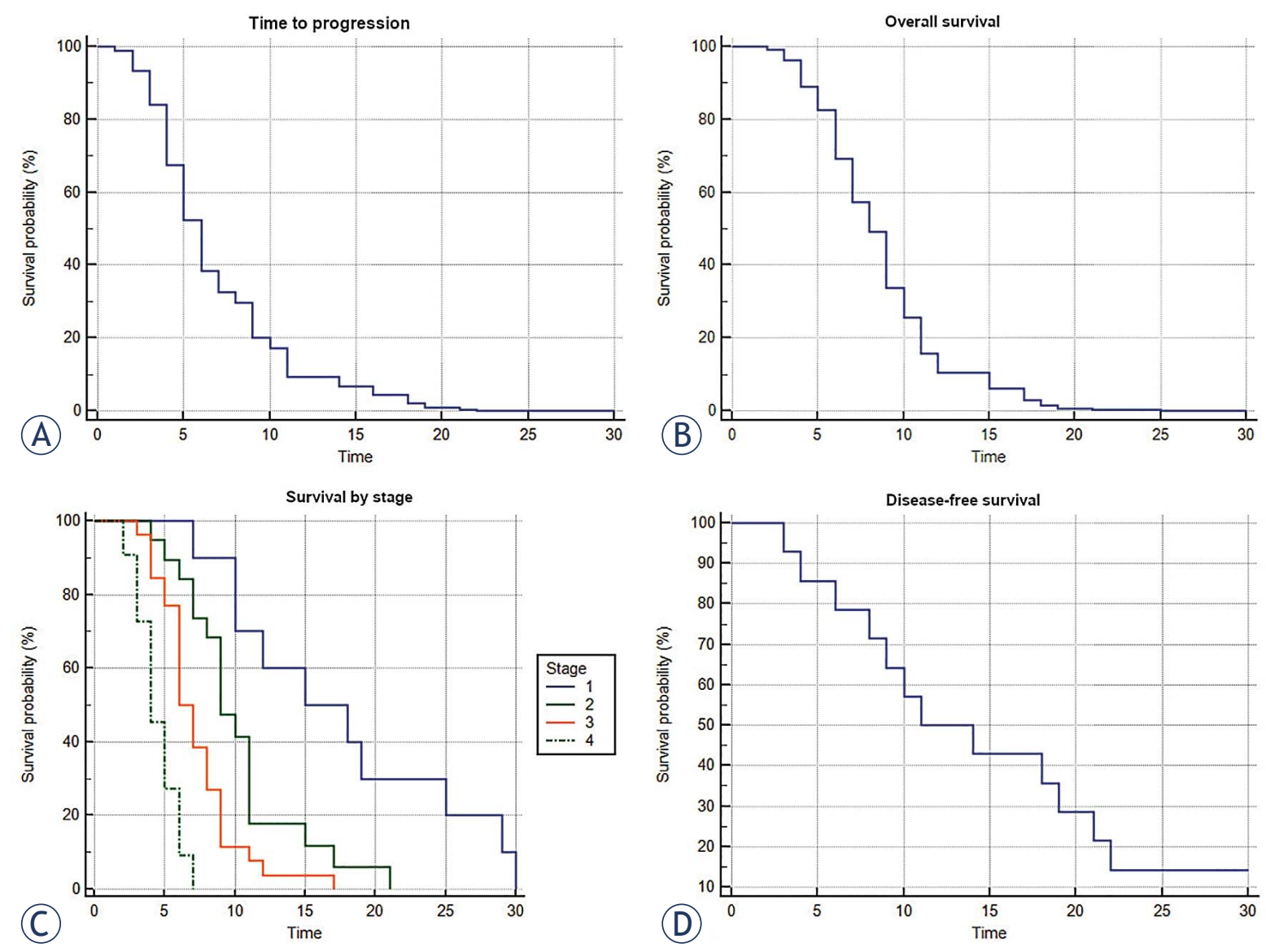

FIGURE 4. (A) Time to progression curve (Cox proportional-hazards regression, $n=67, p<0.0001$ ). (B) Overall survival time (Cox proportional-hazards regression, $n=67, p<0.0001$ ). (C) Survival time by stage (Kaplan-Meier curves of survival, $n=67, p<0.0001$ ). (D) Disease-free survival time (Kaplan-Meier, $\mathrm{n}=14$ ).

\section{Overall survival time}

Median survival time after the treatment was 16.5 (range 4-30) months, 9 (range 4-21) months, 7.5 (range 3-17) months and 4.5 (range 2-7) months, in stage I, II, III and IV, respectively. Two patients remained alive at the end of the study, therefore, in the statistical analysis were censored (Figure 4).

In multivariate analysis for survival time, stage IV $(p=0.0001)$ was a negative predictive factor. On the contrary, stage $I(p=0.0083)$ and the absence of bone involvement $(p=0.0340)$ were predictive factors for longer survival (Table 4).

\section{Cause of death}

Euthanasia was the main cause of death during the course of this study, representing $77.6 \%$ (52) of the deceased patients, where $49.3 \%$ (33) of those euthanasias were performed due to local progression and $28.4 \%$ (19) due to metastatic progression of the disease. Unrelated causes of death reached 17.9\% (12) and finally, 3\% (2) of the overall enrolled patients remained alive by the end of the follow-up period (24 months).

\section{Metastasis}

Among patients with no metastatic lesions at the time of diagnosis, $23.9 \%$ (16) developed new metastasis during subsequent follow-up visits. Patients treated in stage I did not develop new metastasis. For stages II, III and IV, the number of patients that developed metastasis after the first visit was also similar, being $31.6 \%, 36.8 \%$ and $31.6 \%$, respectively.

\section{Quality of life}

Patients in stage I with the tumor in rostral location had no negative impact on their quality of life before or after the treatment. Patients in stages I (with 
TABLE 3. Univariate (Fisher exact test) and multivariate (Logistic regression analysis) test for analyzing the association between OR and different factors

\begin{tabular}{lccc}
\hline Characteristics & $\begin{array}{c}\text { Number of } \\
\text { patients with OR }\end{array}$ & $\begin{array}{c}\text { Univariate } \\
\text { Fisher exact test }\end{array}$ & $\begin{array}{c}\text { OR 95\% Cl Logistic Regression Analysis } \\
\mathbf{p}\end{array}$ \\
\hline Sex (M/F) & $37 / 30$ & $\mathrm{p}=0.602$ & - \\
Location (Rostral/Caudal) & $36 / 31$ & $\mathrm{p}=0.062$ & - \\
Bone Involvement (Yes/No) & $43 / 24$ & $\mathrm{p}=0.026$ & - \\
Metastasis present, excluding lymph nodes (Yes/No) & $10 / 57$ & $\mathrm{p}=0.150$ & - \\
Early-stage (stages I+II/stages III+IV) & $30 / 37$ & $\mathrm{p}=0.00016$ & - \\
\hline
\end{tabular}
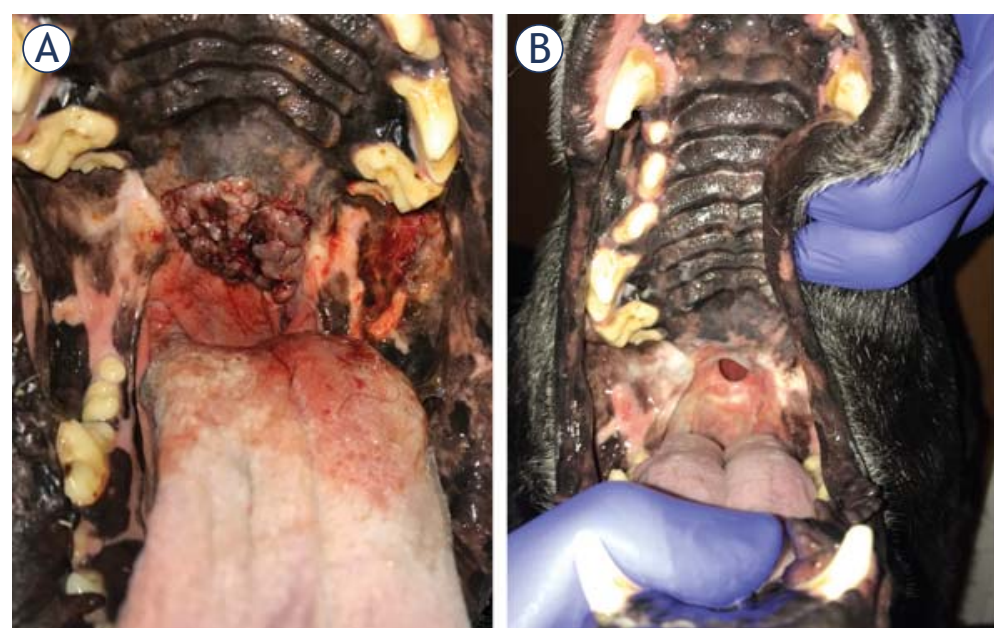

FIGURE 5. (A) Stage $\|$ patient with melanoma in the soft palate, before the treatment. (B) Final response obtained 30 days after third round of ECT. Completely absent tumoral tissue and a fistula can be seen. Quality of life of the patient improved in spite of the fistula, which was asymptomatic.

the tumor in a caudal location), II and III presented the following signs and symptoms: bleeding, accidental biting of the tumor, pain and impossibility to eat adequately. Among the latter, those who obtained a CR or PR after ECT, had an improvement in their quality of life (Figure 5). On the contrary, patients that obtained a SD or PD, did not. Patients in stage IV did not improve their quality of life regardless of the obtained response.

\section{Discussion}

ECT is a very effective local treatment for tumors originating almost any histological type of tissue. In the last few years, the treatment has become readily available in many countries due to its multiple advantages. Among them: high efficacy, low cost and simplicity at time of performing. The aforementioned, combined with the availability of inexpensive devices, made possible its expansion through Latin America, where radiotherapy continuous to be a non-affordable therapeutic alternative. Since often ECT is the only available option, professionals tend to treat indiscriminately, without even taking in consideration if that particular patient is indeed a surgical candidate or not. Therefore, we believe that veterinarians should assess more carefully, in advance, if good results could potentially be achieved with this modality, in order to consider or plan a therapeutic alternative. We think that for an adequate determination who might be considered a good candidate for the treatment, further studies are needed, with the hope that one day ECT could become a first-line therapy for many tumors.

In this study, frequency of breeds, differences in gender predisposition and age at the time of diagnosis were in accordance with different authors. ${ }^{33,34}$ Also, melanomas located in the tongue were rare, as reported by others. ${ }^{35}$ The largest group of patients enrolled in the study were staged II or III. This is probably related to the fact that owners were not able to recognize the characteristic lesions earlier in the onset of the disease, due to the intra oral location of the tumor. Early signs and symptoms were probably unnoticed by the caretakers, but more prominent symptomatology caused by a more advanced stages of the disease was the red flag that made owner seek for medical advice. ${ }^{36} \mathrm{We}$ had a similar prevalence of histological subtypes to the one reported by Ramos-Vara et al., except for a lower incidence of spindle cell type. Also, we had a higher incidence of amelanotic melanomas. ${ }^{33}$

Best local responses were obtained in patients with tumors smaller than $4 \mathrm{~cm}$ in diameter (stages I and II). We came to the conclusion that smaller tumors are easier to treat with ECT than larger ones, not only due to the limitation related to the size of the electrode (lower chances to reach larger tumor extensions), but also because bigger tumors have necrotic areas, and a very inhomogeneous vascularization, affecting the distribution of the drug. We addressed this particular issue in a previous 
TABLE 4. Univariable (Kaplan-Meier) and multivariable (Cox proportional-hazards) analysis of patient-related factors for time to progression and overall survival time. Hazard Ratio and $95 \%$ Confidence interval is reported

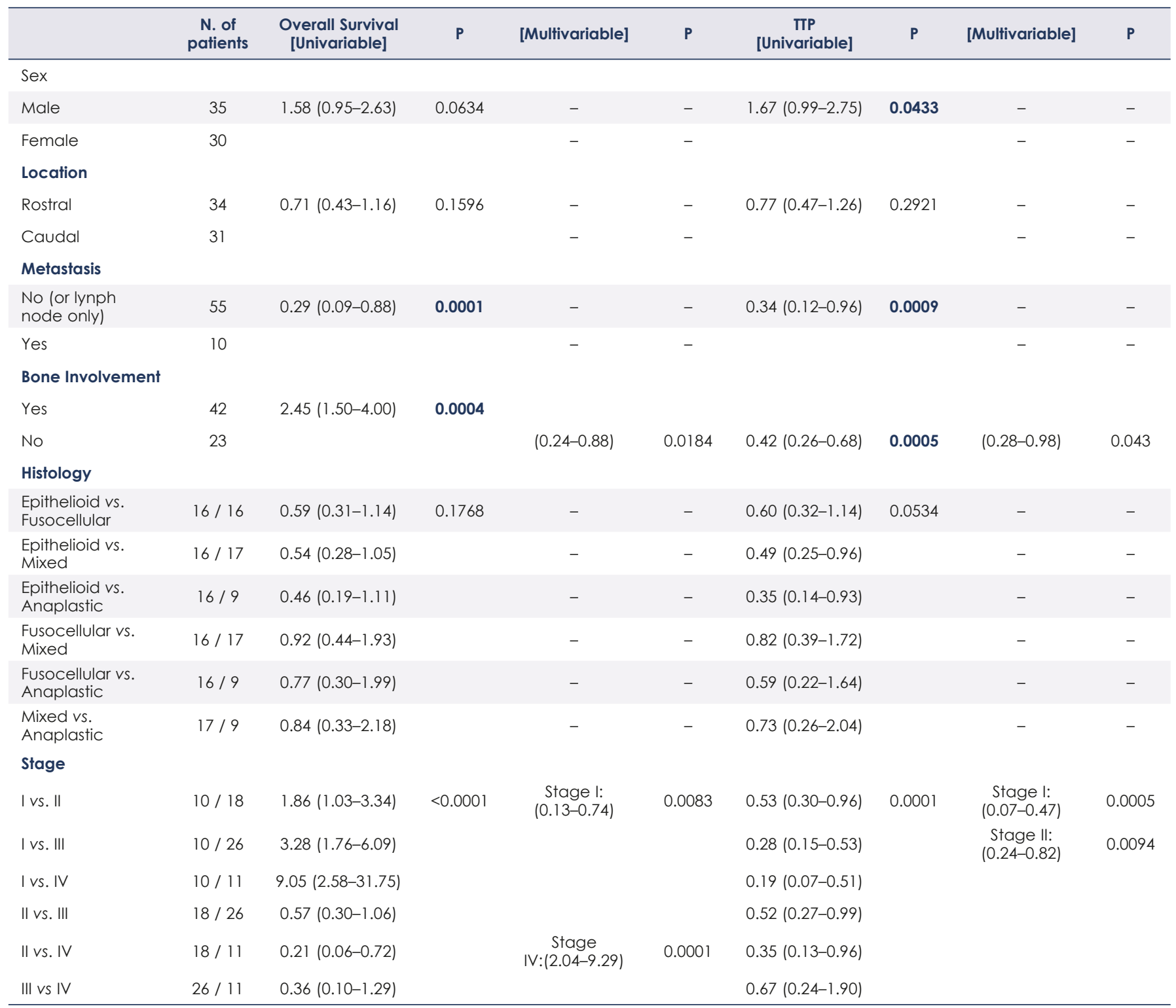

study, by using a combined administration of bleomycin, systemically and locally. ${ }^{37}$

There is still no consensus on when is the best time to perform a second (or subsequent) ECT session. In this work, we considered tumor growth as the main variable to be taken in consideration for that matter. This is based on our $10+$ years of experience performing ECT, and by the fact that we were able to identify four different types of outcomes or evolutions after the first session. In the first one, the most common, the tumor gets steadily smaller until reaching a stable size or disappearing completely. In the second type, the so called "two- times response", the lesion reduces its size up to one point where it stops for some time, resuming shrinkage later until reaching a stable size or disappearing. In the third type, the "apparent unresponsive", it never gets smaller, stays apparently unresponsive to the treatment, but it does not grow and can also remain in that steady state for years. And finally, in the fourth type, "the insufficiently treated", after an initial short period of shrinkage, the tumor starts growing back again. It is known that all these types of evolutions are related to the bleomycin's mechanism of action, which consists in cutting the DNA strands and inducing mitotic 
cell death. If the tumor cell are treated while dividing, it will die. However, if there is a number of quiescent cells not attempting to enter into cell cycle, they will remain alive. This is the reason why this treatment displays selectivity towards dividing cells. ${ }^{9}$ In the light of the above explained, we consider that the only situation that potentially opens the door for a subsequent treatment after the original one is when a regrowth from a previously treated lesion is observed. Otherwise, we can be facing a type two or three evolution.

Absence of bone involvement was associated with longer times to progression and survival. This fact could be attributed partially to how difficult is to insert the electrode inside the bone tissue. Even when the electrode is properly positioned, changes in electric field distribution between bone and tumor tissue can lead to inhomogeneities that will lead to inadequate treatment. ${ }^{38}$ This is in accordance with other authors who treated oral malignant melanoma with surgery plus radiotherapy. They had better results treating small tumors without bone involvement, but they also found that rostral location was associated with better responses. ${ }^{39-41}$ In this work, location of the tumor did not achieve statistical significance to be associated either with local response, time to progression or survival.

If we compare frequency of use between ECT and other treatment modalities, we find that surgery is the most common one performed. The median survival time for dogs with malignant melanoma treated with surgery alone varies from 5 to 11 months..$^{42,43}$ Conservative surgery is only recommended followed by radiotherapy in non-resectable tumors for local control of the disease. Median survival times are similar to the times obtained in this work, and that was expected, since both are local treatments. When surgery is possible and the owner accepts it, we recommend to follow that path, as surgery is a well-established technique in our setting. In these cases, ECT could be used in combination with surgery to extend surgical margins, or even to treat the scar and reduce the recurrence rate (further research is needed to confirm this hypothesis). Recurrence rate after incomplete surgical resection is high $(62-65 \%$ vs. $15-22 \%$ recurrence rate with incomplete and complete resection, respectively), and in those cases we choose to perform ECT as a first-line treatment. ${ }^{44}$

Radiation therapy alone is effective to obtain good local control of the disease, especially with patients that are not suitable for complete surgical excision (the addition of chemotherapy provides no additional benefit). ${ }^{39}$ The response rates are similar regardless of the protocol that was used. Proux et al. achieved 51\% CR, 31\% PR, 16\% SD and $1 \% \mathrm{PD}$. If we compare these results with the ones obtained in this work, we find similar OR rates, but with more $\mathrm{CR}$ when treating with radiotherapy. However, they report a 51\% incidence of new metastasis, while in this work, the incidence of new metastasis was $28.4 \%$. Results of median overall survival time with radiotherapy were 7 months (69 months), also similar to the ones obtained in this work, 7.5 months (2-30 months). ${ }^{39}$ An adequately designed clinical study should be designed performed to compare both treatment modalities.

It is well known that melanoma is an extremely chemotherapy-resistant tumor, and recent studies have been supporting the idea that chemotherapy brings little to no benefits based on the analysis of results obtained after using the aforementioned therapeutic modality. Boria et al..$^{45}$ reported a tumor remission of $18 \%$ in melanoma patients and a median survival time of 4 months.

In relation to lymph node involvement, fineneedle aspiration (FNA) technique is mandatory for patient staging. ${ }^{34}$ Several (FNA) samples obtained from the patients, ended up being positive even after unremarkable lymphadenopathy noticed to palpation at the moment of the exam.

Among the limitations we had to face throughout the making of this study, lack of chances to perform immunohistochemistry in every patient, was probably, one of the most important. Consequently, we had to draw upon the aforementioned technique only when diagnosis of melanoma was not definitive by using simple hematoxylin-eosin stain. Another limitation was imaging. As default, bone involvement was assessed by plain radiographs. CT scan was only performed in selected patients, either due to financial constraints or at times when we felt $\mathrm{X}$-rays were not giving enough information in order to get to a definitive diagnosis.

New immunological approaches are now available in order to improve the results of local therapy. Promising results have been published by Mozillo et al. combining ECT with ipilimumab. The ECT session was performed for local control of the disease after treatment with ipilimumab. It seems that ECT triggered a systemic response, which was obtained in $60 \%$ of the patients. ${ }^{46}$

In the last decade, DNA-based immunotherapy reached important goals in the treatment of infectious diseases and cancer. ${ }^{47}$ In a previous study, we explored the use of ECT in combination with canine IL-2+IL-12 plasmid vector delivered by electroporation, obtaining local control of the disease 
in patients with different histological neoplasms. We observed fever, swelling, and lethargy due to the transfection, which had to be treated with corticosteroids. A single systemic response in lung metastasis was obtained ${ }^{48}$ but further research is needed to study this combination.

Milevoj et al. obtained a $67 \% \mathrm{CR}$ rate in 9 canine patients with an oral malignant melanoma treated with surgery plus electrochemotherapy plus gene electrotransfer with IL-12. They report a declining in regulatory $\mathrm{T}$ cells number that is associated with the treatment. ${ }^{49}$ Cemazar et al. combined ECT with human IL-12 plasmid vector delivered by electroporation and obtained a $72 \% \mathrm{CR}$ rate in spontaneous canine mast cell tumors without side effects. They also observed that the therapy prevented local recurrences and distant metastasis..$^{50}$

ECT is a very appealing treatment modality for combination with immunotherapies, especially with those based on gene electrotransfection. This latter technology allows performing the immunotherapy at a fraction of the cost when compared with traditional immunotherapies. In this sense, low income countries may be the most benefited from the combination of ECT and gene electrotransfer.

In conclusion, ECT is an adequate local treatment modality for canine patients with oral malignant melanoma with lesions of up to $4 \mathrm{~cm}$ in diameter, i.e. stages I and II, and without bone invasion.

\section{Acknowledgments}

F.H.M., S.D.M. and G.R.M. are researchers from Consejo Nacional de Investigaciones Científicas y Técnicas (CONICET). This work is supported by grants from CONICET, www.conicet.gov. ar, (PIP 379/2012/2016 and STAN 534/12), CNR (CNR Project DSB.AD007.072) and Universidad de Buenos Aires, www.uba.ar, (UBACyT 2014/2017). E. Signori was partially supported by CNR-Short Term Mobility Fellowship Prot. $N^{\circ} 72648 / 2019$. The funders had no role in study design, data collection and analysis, decision to publish, or preparation of the manuscript. This manuscript was proof read by PurpleGY.com.

\section{Author contributions statement}

M.N.T. and F.H.M. worked on study design, patient selection and treatment, data analysis and manuscript writing. S.D.M. worked on patient treatment, data analysis and manuscript writing. G.R.M. worked on manuscript writing, group direction and fund administration. E.S. worked on study design, manuscript writing, group coordination and fund administration. All authors reviewed the manuscript.

\section{References}

1. Smedley RC, Spangler WL, Esplin DG, Kitchell BE, Bergman PJ, Ho H-Y, et al. Prognostic markers for canine melanocytic neoplasms: a comparative review of the literature and goals for future investigation. Vet Pathol 2011; 48: 54-72. doi: 10.1177/0300985810390717

2. Harvey HJ, MacEwen EG, Braun D, Patnaik AK, Withrow SJ, Jongeward S. Prognostic criteria for dogs with oral melanoma. J Am Vet Med Assoc 1981; 178: 580-2. PMID: 7263464

3. Bolon B, Calderwood Mays MB, Hall BJ. Characteristics of canine melanomas and comparison of histology and DNA ploidy to their biologic behavior. Vet Pathol 1990; 27: 96-102. doi: 10.1177/030098589002700204

4. Withrow SJ, Thamm D, Vail DM, Liptak J, Page R. Withrow and Macewen's small animal clinical oncology - E-Book. Saunders; 2019.

5. Overly B, Goldschmidt M, Shofer F. Canine oral melanoma: a retrospective study. Vet Cancer Soc Proc 2001; 21: 43. pii: E7. doi: 10.3390/vetsci3010007

6. Nishiya AT, Massoco CO, Felizzola CR, Perlmann E, Batschinski K, Tedardi MV et al. Comparative Aspects of Canine Melanoma. Vet Sci 2016; 3: pii: E7. doi: 10.3390/vetsci3010007

7. Marty M, Sersa G, Garbay JR, Gehl J, Collins CG, Snoj M, et al. Electrochemotherapy - An easy, highly effective and safe treatment of cutaneous and subcutaneous metastases: Results of ESOPE (European Standard Operating Procedures of Electrochemotherapy) study. Eur J Cancer Suppl 2006; 4: 3-13.

8. Kotnik T, Kramar P, Pucihar G, Miklavcic D, Tarek M. Cell membrane electroporation- Part 1: The phenomenon. IEEE Electr Insul Mag 2012; 28:14-23.

9. Mir LM. Bases and rationale of the electrochemotherapy. 11th Mediterranean Conference on Medical and Biomedical Engineering and Computing 2007. p. 622. doi: 10.1007/978-3-540-73044-6_158

10. Tamzali Y, Borde L, Rols MP, Golzio M, Lyazrhi F, Teissie J. Successful treatment of equine sarcoids with cisplatin electrochemotherapy: a retrospective study of 48 cases. Equine Vet J 2012; 44: 214-20. doi: 10.1111/j.20423306.2011.00425.x

11. Campana LG, Miklavčič D, Bertino G, Marconato R, Valpione S, Imarisio I, et al. Electrochemotherapy of superficial tumors - Current status:: Basic principles, operating procedures, shared indications, and emerging applications. Semin Oncol 2019; 46: 173-91. doi: 10.1053/j.seminoncol.2019.04.002

12. Mali B, Jarm T, Snoj M, Sersa G, Miklavcic D. Antitumor effectiveness of electrochemotherapy: a systematic review and meta-analysis. Eur J Surg Oncol 2013; 39: 4-16. doi: 10.1016/j.ejso.2012.08.016

13. Edhemovic I, Brecelj E, Gasljevic G, Marolt Music M, Gorjup V, Mali B, et al. Intraoperative electrochemotherapy of colorectal liver metastases. I Surg Oncol 2014; 110: 320-7. doi: 10.1002/jso.23625

14. Linnert M, Agerholm-Larsen B, Mahmood F, Iversen HK, Gehl J. Treatment of Brain Tumors: Electrochemotherapy. In: Hayat MA, editor. Tumors of the central nervous system. Dordrecht: Springer Netherlands 2014. p. 247-59.

15. Fini $M$, Salamanna F, Parrilli A, Martini L, Cadossi M, Maglio $M$, et al. Electrochemotherapy is effective in the treatment of rat bone metastases. Clin Exp Metastasis 2013; 30: 1033-45. doi: 10.1007/s10585-013-9601-x 
16. Soden DM, Larkin JO, Collins CG, Tangney M, Aarons S, Piggott J, et al. Successful application of targeted electrochemotherapy using novel flexible electrodes and low dose bleomycin to solid tumours. Cancer Lett 2006; 232 300-10. doi: 10.1016/j.canlet.2005.03.057

17. Gehl J, Sersa G, Matthiessen LW, Muir T, Soden D, Occhini A, et al. Updated standard operating procedures for electrochemotherapy of cutaneous tumours and skin metastases. Acta Oncol 2018; 57: 874-82. doi 10.1080/0284186X.2018.1454602

18. Spugnini EP, Baldi A. Electrochemotherapy in Veterinary Oncology: Stateof-the-Art and Perspectives. Vet Clin North Am Small Anim Pract 2019; 49: 967-79. doi: 10.1016/j.cvsm.2019.04.006

19. Rangel MMM, Luz JCS, Oliveira KD, Ojeda J, Freytag JO, Suzuki DO Electrochemotherapy in the treatment of neoplasms in dogs and cats. Austral J Vet Sci 2019; p. 45-51. doi: 10.4067/s0719-81322019000200045

20. Tozon N, Lampreht Tratar U, Znidar K, Sersa G, Teissie J, Cemazar M. Operating procedures of the electrochemotherapy for treatment of tumor in dogs and cats. J Vis Exp 2016. doi: 10.3791/54760.

21. Spugnini EP, Vincenzi B, Citro G, Dotsinsky I, Mudrov T, Baldi A. Evaluation of Cisplatin as an electrochemotherapy agent for the treatment of incompletely excised mast cell tumors in dogs. J Vet Intern Med 2011; 25: 407-11. doi: 10.1111/j.1939-1676.2011.0678.x

22. Kodre V, Cemazar M, Pecar J, Sersa G, Cor A, Tozon N. Electrochemotherapy compared to surgery for treatment of canine mast cell tumours. In Vivo 2009; 23: 55-62.

23. Spugnini EP, Vincenzi B, Citro G, Santini D, Dotsinsky I, Mudrov N, et al. Adjuvant electrochemotherapy for the treatment of incompletely excised spontaneous canine sarcomas. In Vivo 2007; 21: 819-22.

24. Tozon N, Kodre V, Juntes P, Sersa G, Cemazar M. Electrochemotherapy is highly effective for the treatment of canine perianal hepatoid adenoma and epithelioma. Acta veterinaria 2010; 60: 285-302. doi: 10.2298/avb1003285t

25. Maglietti F, Tellado M, Olaiz N, Michinski S, Marshall G. Minimally invasive electrochemotherapy procedure for treating nasal duct tumors in dogs using a single needle electrode. Radiol Oncol 2017; 51: 422-30. doi: 10.1515/ raon-2017-0043

26. Mir LM, Orlowski S, Belehradek J Jr, Paoletti C. Electrochemotherapy potentiation of antitumour effect of bleomycin by local electric pulses. Eur J Cancer 1991; 27: 68-72. doi: 10.1016/0277-5379(91)90064-k

27. Calvet CY, Famin D, André FM, Mir LM. Electrochemotherapy with bleomycin induces hallmarks of immunogenic cell death in murine colon cancer cells. Oncoimmunology 2014; 3: e28131. doi: 10.4161/onci.28131

28. Sersa G, Teissie J, Cemazar M, Signori E, Kamensek U, Marshall G, et al. Electrochemotherapy of tumors as in situ vaccination boosted by immunogene electrotransfer. Cancer Immunol Immunother 2015; 64: 1315-27. doi: $10.1007 / \mathrm{s} 00262-015-1724-2$

29. Sadozai H, Gruber T, Hunger RE, Schenk M. Recent successes and future directions in immunotherapy of cutaneous melanoma. Front Immunol 2017 8: 1617. doi: 10.3389/fimmu.2017.01617

30. Bergman PJ. Canine oral melanoma. Clin Tech Small Anim Pract 2007; 22 55-60. doi: 10.1053/j.ctsap.2007.03.004

31. Campana LG, Clover AJP, Valpione S, Quaglino P, Gehl J, Kunte C, et al. Recommendations for improving the quality of reporting clinical electrochemotherapy studies based on qualitative systematic review. Radiol Oncol 2016; 50: 1-13. doi: 10.1515/raon-2016-0006

32. Lynch S, Savary-Bataille K, Leeuw B, Argyle DJ. Development of a questionnaire assessing health-related quality-of-life in dogs and cats with cancer. Vet Comp Oncol 2011; 9: 172-82. doi: 10.1111/j.1476-5829.2010.00244.x

33. Ramos-Vara JA, Beissenherz ME, Miller MA, Johnson GC, Pace LW, Fard A et al. Retrospective study of 338 canine oral melanomas with clinical, histologic, and immunohistochemical review of 129 cases. Vet Pathol 2000; 37: 597-608. doi: 10.1354/vp.37-6-597

34. Williams LE, Packer RA. Association between lymph node size and metastasis in dogs with oral malignant melanoma: 100 cases (1987-2001). J Am Vet Med Assoc 2003; 222: 1234-6. doi: 10.2460/javma.2003.222.1234

35. Todoroff RJ, Brodey RS. Oral and pharyngeal neoplasia in the dog: a retrospective survey of 361 cases. J Am Vet Med Assoc 1979; 75: 567.

36. Montayeva NS, Kushaliyev KZ, Grabarevic Z. Early symptoms of malignancy in the appearance of melanoma in dogs. Biol Med 2016; 8: 163-16.
37. Maglietti F, Tellado M, Olaiz N, Michinski S, Marshall G. Combined local and systemic bleomycin administration in electrochemotherapy to reduce the number of treatment sessions. Radiol Oncol 2016; 50: 58-63. doi: 10.1515/ raon-2016-0015

38. Miklavcic D, Beravs K, Semrov D, Cemazar M, Demsar F, Sersa G. The importance of electric field distribution for effective in vivo electroporation of tissues. Biophys J 1998; 74: 2152-8. doi: 10.1016/S0006-3495(98)77924-X

39. Proulx DR, Ruslander DM, Dodge RK, Hauck ML, Williams LE, Horn B, et al. A Retrospective analysis of 140 dogs with oral melanoma treated with external beam radiation. Vet Radiol Ultrasound 2003; 44: 352-9. doi: 10.1111/j.1740-8261.2003.tb00468.x

40. Théon AP, Rodriguez C, Madewell BR. Analysis of prognostic factors and patterns of failure in dogs with malignant oral tumors treated with megavoltage irradiation. J Am Vet Med Assoc 1997; 210: 778-84.

41. Blackwood L, Dobson JM. Radiotherapy of oral malignant melanomas in dogs. J Am Vet Med Assoc 1996; 209: 98-102.

42. MacEwen EG, Kurzman ID, Vail DM, Dubielzig RR, Everlith $K$, Madewell $B R$, et al. Adjuvant therapy for melanoma in dogs: results of randomized clinical trials using surgery, liposome-encapsulated muramyl tripeptide, and granulocyte macrophage colony-stimulating factor. Clin Cancer Res 1999; 5: 4249-58.

43. Freeman KP, Hahn KA, Harris FD, King GK. Treatment of dogs with oral melanoma by hypofractionated radiation therapy and platinum-based chemotherapy (1987-1997). J Vet Intern Med 2003; 17: 96-101. doi: 10.1892/08916640(2003)017<0096:todwom>2.3.co;2

44. Schwarz PD, Withrow SJ, Curtis CR. Partial maxillary resection as a treatment for oral cancer in 61 dogs. J Am Anim Hosp Assoc 1991; 27: 617-24.

45. Boria PA, Murry DJ, Bennett PF, Glickman NW, Snyder PW, Merkel BL, et al. Evaluation of cisplatin combined with piroxicam for the treatment of oral malignant melanoma and oral squamous cell carcinoma in dogs. J Am Vet Med Assoc 2004; 224: 388-94. doi: 10.2460/javma.2004.224.388

46. Mozzillo N, Simeone E, Benedetto L, Curvietto M, Giannarelli D, Gentilcore $G$, et al. Assessing a novel immuno-oncology-based combination therapy: Ipilimumab plus electrochemotherapy. Oncoimmunology 2015; 4: e1008842. doi: 10.1080/2162402X.2015.1008842

47. Chiarella $P$, Fazio VM, Signori E. Electroporation in DNA vaccination protocols against cancer. Curr Drug Metab 2013; 14: 291-9. doi: 10.2174/1389200211314030004

48. Maglietti F, Michinski S, Emanuela S, Tellado M, Marshall G. Electrochemotherapy immune response enhancement by gene electrotransfer using IL-2 and IL-12 genes in canine patients. Eur J Cancer 2016; 61: S210. doi: 10.1016/s0959-8049(16)61741-0

49. Milevoj N, Lampreht Tratar U, Nemec A, Brožič A, Žnidar K, Serša G, et al. A combination of electrochemotherapy, gene electrotransfer of plasmid encoding canine IL- 12 and cytoreductive surgery in the treatment of canine oral malignant melanoma. Res Vet Sci 2019; 122: 40-9. doi: 10.1016/j. rvsc.2018.11.001

50. Cemazar M, Ambrozic Avgustin J, Pavlin D, Sersa G, Poli A, Krhac Levacic A, et al. Efficacy and safety of electrochemotherapy combined with peritumoral IL-12 gene electrotransfer of canine mast cell tumours. Vet Comp Oncol 2016; 15: 641-54 doi: 10.1111/vco.12208 